\title{
Anterior Cruciate Ligament Rehabilitation and Return to Sport: How Fast Is Too Fast?
}

\author{
Kristen Waldron, P.T., D.P.T., S.C.S., Matthew Brown, P.T., D.P.T, S.C.S, C.S.C.S., \\ Ariana Calderon, P.T., D.P.T, and Michael Feldman, M.D.
}

\begin{abstract}
This article summarizes the benefits and limitations of various approaches of anterior cruciate ligament (ACL) rehabilitation, more specifically a conservative or traditional rehabilitation approach versus a more accelerated approach. The conservative model is considered one with a return to sport at 9 months or later with more time-based criteria, and an accelerated approach is defined as one with a goal of return to sport by 6 months. Although there are some similarities between the 2 types of rehabilitation, key differences exist and will be highlighted. Additionally, we discuss a criteriabased return-to-sport model that we favor. Level of Evidence: V, expert opinion.
\end{abstract}

$\mathbf{T}$ There are an estimated 200,000 anterior cruciate ligament (ACL) injuries per year. ${ }^{1}$ ACL reconstruction (ACLR) is a common treatment strategy for this injury, as many patients have the goal of returning to competitive sport. ${ }^{2}$ With this type of procedure, $\sim 80 \%$ patients return to some type of sport, but only $65 \%$ return to preinjury levels of participation, and only $55 \%$ return to competitive sport. ${ }^{2}$ With this in mind, it is important for the sports medicine team to find the optimal rehabilitation approach to maximize the number of patients achieving their preferred return-to-sport goals.

Over the last few years, strictly time-based rehabilitation protocols have started to be replaced by criteriabased rehabilitation protocols. ${ }^{1}$ The return-to-sport decision should consider a multitude of factors, including concomitant injuries, objective physical examination data, functional return-to-sport testing, psychological readiness, activity-specific demands, and

From BodyCentral Physical Therapy, Tucson, Arizona, U.S.A. (K.W., M.B., A.C.); and the University of Arizona College of Medicine, Banner University Medical Center, Tucson, Arizona, U.S.A. (M.F.).

The authors report the following potential conflicts of interest or sources of funding: M.F. reports associate editor, Journal of Arthroscopy. Full ICMJE author disclosure forms are available for this article online, as supplementary material.

Received August 2, 2021; accepted October 9, 2021.

Address correspondence to U.S.A. E-mail:

(C) 2021 THE AUTHORS. Published by Elsevier Inc. on behalf of the Arthroscopy Association of North America. This is an open access article under the CC BY-NC-ND license (http://creativecommons.org/licenses/by-nc-nd/4.0/).

2666-061X/211106

https://doi.org/10.1016/j.asmr.2021.10.027 consideration of biological tissue healing. ${ }^{3}$ However, there is still some debate as to the speed of rehabilitation to achieve these return-to-sport goals. Typically, 2 rehabilitation approaches are followed: a conservative or traditional approach, which generally aims for return to sport after 9 to 12 months, and an accelerated approach, which would return patients to sport within 6 months of reconstruction. ${ }^{4}$ The purpose of this review is to discuss each of these approaches, discuss their positives and negatives, and determine when it is appropriate to use one rehabilitation approach over the other.

\section{Conservative/Traditional Approach}

There is no established conservative protocol for ACL rehabilitation, but there is a general consensus to prolong the timeline for return to sport to allow adequate healing. Several studies have considered traditional or conservative ACL rehabilitation to be old protocols that involve longer immobilization of up to 6 to 8 week,s followed by a progression of weightbearing as tolerated. For this particular literature review, conservative protocol will be defined as returning to sport after 9 months or later and basing criteria for certain tasks on time rather than objective testing. ${ }^{5}$

In a literature review of 6 clinical practice guidelines (CPGs), recommendations for rehabilitation vary regarding the use of an accelerated approach versus a conservative approach. ${ }^{2,6-11}$ of the articles we reviewed, 3 gave unclear guidelines on accelerated versus conservative protocols. ${ }^{2}$ However, research has shown deficits in strength, balance, joint forces, and kinesthesia anywhere from 6 months to 2 years after 
surgery. ${ }^{1}$ This may lead some providers to choose a more conservative approach to further address these deficits before return to full activity.

With a conservative rehabilitation approach, considerations of the biological factors of tissue healing play into determining the timeline for return to sport. The graft tissue undergoes necrosis, revascularization, and remodeling, leading many researchers to recommend return to full activity after 9 to 12 months. ${ }^{12}$ Revascularization and ligamentization typically occurs over a 12-month period for allografts; however, vascularity and fiber pattern may normalize after 6 to 12 months. ${ }^{12}$

Initial rehabilitation exercises are somewhat similar between conservative and accelerated approaches and strive for similar outcomes over the long term. Rehabilitation's goals are to normalize range of motion (ROM), improve quadricep activation and lower extremity strength, and restore normal biomechanics, as deficits in these areas can negatively impact lower extremity function and performance. ${ }^{13}$ The overall goal is for the knee to achieve normalized joint homeostasis, neuromuscular control, strength, and proprioception; however, this can take $\leq 2$ years in some patients. ${ }^{14}$ With a traditional approach, patients go through isokinetic testing at 6 months and are typically released to full activity at 9 to 12 months, seeing that full ROM and all functional progressions have been achieved. ${ }^{15}$ Although using purely time-based criteria may not be appropriate, there is some evidence that reinjury incidence is reduced by $51 \%$ per month for each month that return to sport is delayed (at months 5 to 9 postoperatively). ${ }^{14,16}$ This supports the use of a more conservative model and that there is a minimum time frame required to allow appropriate graft maturation.

The conservative rehabilitation approach for ACLR has been effective, and research continues to show that the conservative approach has outcomes similar to an accelerated approach. Some evidence supporting the conservative approach shows that reinjury rate reduction $\leq 9$ months can be beneficial for many reasons, including increased time to build strength, graft maturation and healing, neuromuscular control, and conditioning for sport.

\section{Accelerated Approach}

After ACLR, an accelerated rehabilitation program includes early unrestricted motion, immediate weightbearing as tolerated, eliminating immobilizing braces, ${ }^{17}$ and a patient-dependent aim to return to high-level physical activities or sport within 6 months postoperatively. ${ }^{17}$ The increase of knee laxity in anterior, posterior, and coupled rotation in both conservative and accelerated rehabilitation has been shown to have no significant difference at 2 year follow-up, demonstrating that immediate motion and weightbearing are safe for the graft. ${ }^{18}$ In this context, return to sport is defined as "achieving the preinjury level of sports participation as defined by the same type, frequency, intensity, and quality of performance as before injury."14

The aim of an accelerated rehabilitation program, like that of the traditional model, is to restore prior level of function, strength, ROM, proprioception, neuromuscular control, and joint stability and decrease kinesiophobia while maintaining the integrity of the given graft. Some important advantages to consider when taking an accelerated approach are decreased cost, earlier return to sport, and earlier recovery of objective measures such as ROM and strength. ${ }^{4}$ When considering each patient, especially in the active population, quality of life is drastically changed by the loss of lower extremity function, increasing the chance for the development of depression. The type of graft is a consideration when preparing any rehabilitation program, but more research must be done to analyze the influence of graft type on accelerated rehabilitation.

Comparing the accelerated protocol to conservative patients in both elite athletes and nonathletes, Feyzioğlu et al. ${ }^{19}$ found that accelerated rehabilitation decreased disability, depression, and pain; increased functionality; and improved quality of life. In the process of returning to sport, specific criteria, rather than timeline-based progression, should be used. This requires the completion of objective tests and measures to progress to the next phase. Multiple tests and measures, both qualitative and quantitative, should be used together to determine readiness for return to participation and ultimately return to sport. Tests shown to be of significance include ROM, presence of effusion, quadricep and hamstring strength, joint laxity, limb symmetry index $(>90 \%)$, subjective reports through outcome measures, single-limb hop tests, balance, and a measure of quality of movement. More research is needed to determine the best testing battery. ${ }^{14}$

Ideally, patients will progress through a structured plan of care that allows them to participate in sportspecific activity along a continuum of clearance after completing required milestones. Special considerations must be made for each individual, considering physical performance criteria, sport demand, mental readiness, lesion type, surgical technique, genetics, and other factors. Not every person is a candidate to complete an accelerated protocol, and clinical experience and judgment must be used with each case. A multidisciplinary approach with open communication allows for the surgeon and rehabilitation team to decide on a unique course of action for each patient. Overall, an accelerated rehabilitation protocol does not contribute to increased laxity and can grant the desired quality of life sooner than conservative measures. 


\section{Conservative Versus Accelerated}

With both approaches being considered, what is the best approach for the patient? There continue to be variations of exercises included in ACL rehabilitation protocols, as well as time requirements. ${ }^{20}$ There are a multitude of factors to consider with rehabilitation choice, such as type of sport demands, patient characteristics, anatomic variations, graft type, and presence of concomitant injuries. ${ }^{3}$

The question that has led to this research is whether there are differences that can be measured between accelerated or conservative ACL rehabilitation that include injury risk, joint laxity, strength, return to prior level of function, and return to sport, as well as the speed of return to sport. Hiranyakumar and Karthik ${ }^{21}$ looked at accelerated versus traditional rehabilitation and found that in an accelerated program, patients were able to recover strength earlier: at 6 months, strength was significantly different, but no difference was found at 9 months. Similarly, Hedbys ${ }^{22}$ looked at quadricep strength and quadricep limb symmetry index and found a tendency for higher measures in both for accelerated rehabilitation, but the differences were not clinically significant between accelerated and conservative groups at 4, 6, 8, 12, and 24 months. In 2021, Cristiani et al. ${ }^{23}$ studied how standard or accelerated protocols affected different graft types by looking at the strength of quadriceps and hamstrings as well as the single-leg hop test at 4, 6, 8, 12, and 24 months. They found no differences statistically between standard and accelerated rehabilitation for all measures at all time points. ${ }^{23}$ Gupta et al. ${ }^{24}$ looked at knee laxity, International Knee Documentation Committee (IKDC) scores, Knee Injury and Osteoarthritis Outcome Score (KOOS), and single-leg hop tests between accelerated and standard protocols. They found no difference found in knee laxity, activity level, patient satisfaction, or functional performance between the 2 protocols, but found statistically significant differences in IKDC and KOOS at 3 and 6 months. ${ }^{24}$

There is a tendency toward the accelerated protocol for improving strength, but most research has not shown a statistically significant difference in objective findings. Further research should continue to look at reinjury rates, functional measures, and strength at 24 months and beyond to compare these 2 protocols. Some positives of a conservative approach include increased healing time, less stress to the graft earlier in the rehabilitation timeline, and possibly a decrease in injury or reinjury risk associated with delayed return to sport. Retear rates for $\leq 2$ years postsurgery are much higher than the profession would like both in ipsilateral and contralateral limbs, from $6 \%$ to $31 \%$, and maybe we are making the wrong choice in returning athletes too early to sport. $^{25}$ Some limitations with a conservative approach are decreased strength gains earlier in rehabilitation, possible lack of compliance with the rehabilitation program and timeline, longer return to sport (which can impact people financially and mentally), and increased cost of health care. On the other side, advantages for an accelerated approach are quicker strength gains and earlier return to sport, which can impact scholarships, mental health, professional opportunities, and cost of health care. Disadvantages of an accelerated approach are possible increased likelihood for retear, potential insufficient time for graft maturation and healing, the possibility of other injuries due to rushing back into sport, and decreased time for return to prior levels of performance including conditioning, strength, and neuromuscular control.

\section{Criteria-Based Return-to-Sport Model}

As noted, each athlete is an individual, and at times an accelerated time frame may be more appropriate, such as with professional athletes or those who have good baseline strength before surgery. Similarly, at times a more conservative time frame is preferred, such as with allograft reconstruction (allograft is a weaker material than autograft), hypermobility, skeletally immature patients, concomitant injuries, or previous ACL repairs. Across the board, the biggest takeaway is that the multidisciplinary health care team should be looking at a criteria-based return to sport, rather than just strictly time since surgery. Although the initial phases will be similar with both approaches, it is important to have criteria that the patient meets to be cleared to return to sport. This must include more than just normalized ROM, absence of pain, and absence of swelling/effusion. In addition to the battery of testing, patient-reported outcome measures should be tracked throughout the rehabilitation process to monitor patient beliefs, psychological readiness, kinesiophobia, etc. $^{26-28}$

Generally, our athletes are going to initiate return to higher-level activity or the beginning phases of return to sport after 12 to 16 weeks. ${ }^{26-28}$ To enter this phase of rehabilitation, the athlete must have met the following criteria: full knee ROM; no pain or swelling; limb symmetry of $75 \%$ to $85 \%$ with handheld dynamometry, isokinetic testing, or 10-repetition maximum testing (depending on available equipment) of the quadriceps, hamstrings, gluteus maximus, and gluteus medius; 10 repetitions of a single-leg squat through $60^{\circ}$ ROM and/or a single-leg stork balance test; and Y-balance test with $90 \%$ limb symmetry. Goals of this phase of rehabilitation include continued advanced strengthening, return to running, initiation of a plyometric program, initiation of an agility program, and incorporation of some sport-specific movements. ${ }^{26-28}$ With all of these programs, athletes will start with submaximal 
efforts, beginning with straight plane movements and 2-leg movements.

Athletes should be assessed for quantitative as well as qualitative outcomes. With landing mechanics, the rehabilitation team is looking to minimize knee valgus, hip internal rotation, hip drop, heavy or stiff landings, and loss of balance. ${ }^{26}$ As the athlete progresses through the initial phases of return to sports, retesting of objective measures should occur at least every 4 to 6 weeks, with the athlete progressing to multiplanar movements and maximal-effort plyometrics once $85 \%$ to $90 \%$ limb symmetry has been achieved with strength testing and $80 \%$ to $90 \%$ limb symmetry with hop testing. ${ }^{26-28}$ At that point, the athlete should also start return-to-play progression: first agility and plyometric drills on the field, then noncontact practice, progressing to contact or full practice, and ending with return to full game play. ${ }^{27}$

\section{Conclusions}

At this point, there is no universally accepted timeline for return to sport, but evidence shows benefits to both conservative and accelerated approaches, depending on the specific patient's needs and the demands of the sport. Although no one specific set of return-to-play guidelines is considered the gold standard, there is growing evidence that return-to-play decisions should be multidisciplinary and criteria-based in nature and consider a multitude of factors including objective examination data, a return-to-sport or functional testing battery, biological tissue healing, psychological readiness, demands of the sport, and patient-specific factors such as concomitant injuries and comorbidities. Further research needs to be done to determine the optimal sequencing and passing criteria for return to play.

\section{References}

1. Cavanaugh JT, Powers M. ACL Rehabilitation progression: Where are we now? Curr Rev Musculoskel Med 2017;10:289-296.

2. Andrade R, Pereira R, van Cingel R, Staal JB, EspregueiraMendes J. How should clinicians rehabilitate patients after ACL reconstruction? A systematic review of clinical practice guidelines (CPGs) with a focus on quality appraisal (AGREE II). Br J Sports Med 2020;54:512-519.

3. Rothrauff BB, Karlsson J, Musahl V, Irrgang JJ, Fu FH. ACL consensus on treatment, outcome, and return to sport. Knee Surg Sports Traumatol Arthrosc 2020;28: 2387-2389.

4. Silva F, Ribeiro F, Oliveira J. Effect of an accelerated ACL rehabilitation protocol on knee proprioception and muscle strength after anterior cruciate ligament reconstruction. Arch Exerc Health Dis 2012;3:139-144.

5. Indorato D, Sturgil R. An assessment of rehabilitation protocols following anterior cruciate ligament reconstruction: A systematic review. Rehab Process Outcome 2016;5. RPO.S40054.
6. Shea KG, Carey JL. Management of anterior cruciate ligament injuries: Evidence-based guideline. J Am Acad Orthop Surg 2015;23:el-e5.

7. Wright RW, Haas AK, Anderson J, et al. Anterior cruciate ligament reconstruction rehabilitation: MOON guidelines. Sports Health 2015;7:239-243.

8. Logerstedt DS, Scalzitti D, Risberg MA, et al. Knee stability and movement coordination impairments: Knee ligament sprain revision 2017. J Orthop Sports Phys Ther 2017;47: $\mathrm{Al}-\mathrm{A} 47$.

9. van Melick N. Return to play after anterior cruciate ligament reconstruction in pivoting athletes [Thesis]. Alblasserdam, Netherlands: Ridderprint. Available at, https:// hdl.handle.net/2066/201196. Accessed July 26, 2021.

10. Arroll B, Robb G, Sutich E. The diagnosis and management of soft tissue knee injuries: Internal derangements. New Zealand Guidelines Group. 2003;1-99. Available at, www.acc.co.nz/assets/provider/662a2a7fb4/accl331 -softknee-injuries.pdf.

11. Meuffels DE, Poldervaart MT, Diercks RL, et al. Guideline on anterior cruciate ligament injury. Acta Orthop 2012;83: 379-386.

12. Falconiero RP, Distefano VJ, Cook TM. Revascularization and ligamentization of autogenous anterior cruciate ligament grafts in humans. Arthroscopy 1998;14:197-205.

13. Bien DP, Dubuque TJ, Bien D. Considerations for late stage ACL rehabilitation and return to sport to limit reinjury risk and maximize athletic performance. Int $J$ Sports Phys Ther 2015;10:256-271.

14. Meredith SJ, Rauer T, Chmielewski TL, et al. Return to sport after anterior cruciate ligament injury: Panther Symposium ACL Injury Return to Sport Consensus Group. Orthop J Sports Med 2020;8:1-11.

15. DeCarlo MS, Shelbourne KD, McCarroll JR, Rettig AC. Traditional versus accelerated rehabilitation following ACL reconstruction: A one-year follow-up. J Orthop Sports Phys Ther 1992;15:309-316.

16. Grindem H, Snyder-Mackler L, Moksnes H, Engebretsen L, Risberg MA. Simple decision rules can reduce reinjury risk by $84 \%$ after ACL reconstruction: The Delaware-Oslo ACL cohort study. Br J Sports Med 2016;50: 804-808.

17. Janssen RPA, van Melick N, van Mourik JBA, Reijman M, van Rhijn LW. ACL reconstruction with hamstring tendon autograft and accelerated brace-free rehabilitation: A systematic review of clinical outcomes. BMJ Open Sport Exerc Med 2018;4:e000301.

18. Beynnon BD, Johnson RJ, Naud S, et al. Accelerated versus nonaccelerated rehabilitation after anterior cruciate ligament reconstruction: A prospective, randomized, double-blind investigation evaluating knee joint laxity using roentgen stereophotogrammetric analysis. Am J Sports Med 2011;39:2536-2548.

19. Feyzioğlu Ö, Öztürk Ö, Sirmen B, Muğrabi S. Does an accelerated program give equivalent results in both elite athletes and nonathletes? J Sport Rehab 2020;29:572-577.

20. Makhni EC, Crump EK, Steinhaus ME, et al. Quality and variability of online available physical therapy protocols from academic orthopaedic surgery programs for anterior cruciate ligament reconstruction. Arthroscopy 2016;32: 1612-1621. 
21. Hiranyakumar S, Karthik MN. Functional outcome of accelerated rehabilitation in arthroscopic anterior cruciate ligament reconstruction with semitendinosis and gracilis graft. Int J Sci Study 2015;3:40-44.

22. Hedbys F. A comparison between accelerated and standard rehabilitation regime on strength outcomes up to 24 months post-operative following ACL-reconstruction [Dissertation]. 2019. Available at, http://uu.diva-portal. org/smash/record.jsf?pid=diva2\%3A 1346525 \&dswid= 5830 .

23. Cristiani R, Mikkelsen C, Wange P, Olsson D, Stålman A, Engström B. Autograft type affects muscle strength and hop performance after ACL reconstruction. A randomised controlled trial comparing patellar tendon and hamstring tendon autografts with standard or accelerated rehabilitation. Knee Surg Sports Traumatol Arthrosc 2021;29: 3025-3036.

24. Gupta PK, Acharya A, Mourya A, Ameriya D. Role of accelerated rehabilitation versus standard rehabilitation following anterior cruciate ligament reconstruction using hamstring graft. J Arthrosc Joint Surg 2017;4: 89-93.

25. Paterno Mv, Rauh MJ, Schmitt LC, Ford KR, Hewett TE. Incidence of second ACL injuries 2 years after primary ACL reconstruction and return to sport. Am J Sports Med 2014;42:1567-1573.

26. Gundersen Health System. ACL Reconstruction Rehabilitation Program. 2019. Available at, https://www. gundersenhealth.org/app/files/public/80a06813-e491-40 02-a90a-c46bb2c29cbe/Sports-Medicine-Protocol-ACLReconstruction.pdf. Accessed July 26, 2021.

27. Massachusetts General Hospital Sports Medicine. Rehabilitation Protocol for ACL Reconstruction. 2018. Available at, https://www.massgeneral.org/assets/MGH/pdf/orth opaedics/sports-medicine/physical-therapy/rehabilitationprotocol-for-ACL.pdf. Accessed July 26, 2021.

28. Bizzini M, Hancock D, Impellizzeri F. Suggestions from the field for return to sports participation following anterior cruciate ligament reconstruction: Soccer. J Orthop Sports Phys Ther 2012;42:304-312. 